\title{
Evaluation method of clean power grid development mode
}

\author{
Bingqi Jiao ${ }^{1,2, *}$, Zhicheng $\mathrm{Xu}^{1,2}$, Kuan Zheng ${ }^{1,2}$, Xiaoqing Yan ${ }^{1,2}$, Junshu Feng ${ }^{1,2}$ \\ ${ }^{1}$ State Grid Energy Research Institue Co., Ltd., Changping District, Beijing 102209, China \\ ${ }^{2}$ State Grid Energy and Power System Planning Research Laboratory, Changping District, Beijing 102209, China
}

\begin{abstract}
In response to the climate change, it has been becoming the consensus of most countries in the world to accelerate the development of a high proportion of clean energy. The power grid is the core to support the development of a high proportion of clean energy, and the key is to accelerate the construction of clean power grids. This paper focuses on the main characteristics of clean power grid construction, and proposes a set of clean power grid development evaluation indicators including 5 first-level indicators and 19 second-level indicators and an analysis method based on radar charts. Taking China Power Grid as an example, this paper analyzes in detail the characteristics of the medium and long-term development mode of clean power grids and the relative change trends of specific indicators, and discusses the key links and potential problems that need attention in the development of clean power grids.
\end{abstract}

\section{Introduction}

In September 2020, Chinese President Xi Jinping solemnly promised at the General Debate of the 75th United Nations General Assembly that China will scale up its Intended Nationally Determined Contributions by adopting more vigorous policies and measures, and aim to have $\mathrm{CO} 2$ emissions peak before 2030 and achieve carbon neutrality before 2060 [1]. Some studies have shown [2] that accelerating the development of clean power is the key to promoting energy transition, and achieving carbon emissions peak and carbon neutrality.

At present, some scholars have carried out relevant researches on the evaluation of the development of clean power grids. Wang et al. [3] focused on the needs of smart grid stakeholders and constructed a smart grid evaluation index system with two levels of strategic index set and process index set. Wang et al. [4] selected 8 key indicators that are universal and can comprehensively reflect the overall development and operation and management level of the power grid, built a world-wide key indicator database to quantitatively evaluate the power grid and determine the interval of first-class level of the international power grid. Liu et al. [5] proposed quantifiable technical and economic indicators of renewable energy systems, which include power generation, economic costs, incomes and carbon emissions. Yang et al. [6] proposed a comprehensive evaluation standard including 22 indicators involving energy, economy, environment, technology and society. Existing researches focused on the power grid or clean energy itself, and the constructed evaluation index system cannot effectively describe the characteristics of

\footnotetext{
* Corresponding author: jiaobingqi@sgeri.sgcc.com.cn
}

the power grid supporting the development of clean energy.

This paper aims at the development prospect of clean power grids supporting the integration of a high proportion of clean energy, and proposes a set of clean power grid development evaluation indicators. First, comprehensively considering the internal and external factors of power grid development, we research and build a set of evaluation indicators focusing on security, interconnection, smart interaction, low carbon and economy, and flexibility, and give a radar chart analysis process. Secondly, taking China Power Grid as an example, the proposed method is used to analyze the characteristics of the medium and long-term development mode of the clean power grid and compare the specific indicators. Finally, a summary of this paper is made.

\section{Evaluation indicators of clean power grid development mode}

In accordance with the development trend of energy transition, considering the factors such as electrification trend, power demand characteristics, energy resource conditions, resource development technology and economy, power grid technology development, etc., the paper builds a set of clean grid development model evaluation indicators as shown in Table 1. The indicators are composed of five classes including security, interconnection, smart interaction, low carbon and economy, and flexibility. The class of security mainly includes the safe power supply capability, topology robustness, anti-risk capability and power supply 
reliability. The class of interconnection mainly includes the ability to configure power resources in a wide area, the ability to integrate diverse generation resources, and the ability to integrate with other energy systems. The class of smart interaction includes the level of scheduling intelligence and the friendliness of source-network-load interaction. The class of low carbon and economy mainly includes the climate friendliness, the level of comprehensive energy utilization, the level of non-hydro renewable energy utilization, the level of clean energy utilization, the utilization level of transmission and distribution assets, and the level of line loss rate. The class of flexibility mainly includes the level of flexible power supply, the level of demand-side response, the level of grid operation flexibility, and the level of electricity marketization.

Table 1. Evaluation indicators of the clean power grid development mode

\begin{tabular}{|c|c|}
\hline First-level indicators & Second-level indicators \\
\hline \multirow{4}{*}{ Security } & Safe power supply capability \\
\hline & Topology robustness \\
\hline & Anti-risk ability \\
\hline & Power supply reliability \\
\hline \multirow{3}{*}{ Interconnection } & $\begin{array}{l}\text { Ability to configure power resources } \\
\text { in a wide area }\end{array}$ \\
\hline & $\begin{array}{l}\text { Ability to integrate diverse } \\
\text { generation resources }\end{array}$ \\
\hline & $\begin{array}{l}\text { Ability to integrate with other } \\
\text { energy systems }\end{array}$ \\
\hline \multirow[b]{2}{*}{ Smart interaction } & Level of scheduling intelligence \\
\hline & $\begin{array}{l}\text { Friendliness of source-network-load } \\
\text { interaction }\end{array}$ \\
\hline \multirow{6}{*}{$\begin{array}{l}\text { Low carbon and } \\
\text { economy }\end{array}$} & Climate friendliness \\
\hline & $\begin{array}{l}\text { Level of comprehensive energy } \\
\text { utilization }\end{array}$ \\
\hline & $\begin{array}{l}\text { Level of non-hydro renewable } \\
\text { energy utilization }\end{array}$ \\
\hline & Level of clean energy utilization \\
\hline & $\begin{array}{l}\text { Utilization level of transmission and } \\
\text { distribution assets }\end{array}$ \\
\hline & Level of line loss rate \\
\hline \multirow{4}{*}{ Flexibility } & Level of flexible power supply \\
\hline & Level of demand-side response \\
\hline & Level of grid operation flexibility \\
\hline & Level of electricity marketization \\
\hline
\end{tabular}

\section{Evaluation method}

In this paper, the radar chart analysis method is used to evaluate the evaluation indicators of the clean power grid development mode, which is a commonly used method in comprehensive evaluation. It is especially suitable for global and overall evaluation of the objects described by a multi-attribute system structure. It can compare and analyze multiple indicators at the same time and study the changes of the same indicator in different periods. The specific steps include: determine the analysis index, collect the index data, draw the radar chart, and give the qualitative evaluation result.

\section{Case study}

The proposed evaluation method is applied to analyze China's clean grid development mode under the vision of a high proportion of clean energy. This paper selects the baseline level year of 2020 and the two long-term level years of 2030 and 2060, and scores the secondary-level indicators in Table 1 one by one using the expert scoring method. The radar chart of the evaluation of China's clean power grid development mode in the medium and long term is shown in Figure 1.

For the security of clean power grid, although the value of the indicator of anti-risk ability is gradually improving, the value is lower than the other three indicators related to security due to the changes of internal and external security risk factors. At this stage, the safety of the power grid is mainly affected by natural disasters and equipment failures, while cyber risks will also become an important factor in the future. The indicators of safe power supply capability, topology robustness, and power supply reliability are currently at a relatively high level, and their future changes will be relatively small.

For the interconnection of clean power grid, the three indicators have greater room for improvement in the future. Taking into account the development expectations of inter-regional transmission channels, the ability to configure power resources in a wide area will be mainly improved before 2030 . With the accelerated development of centralized and distributed new energy after 2030, and the gradual maturity of multi-energy complementarity solutions, the abilities to integrate diverse generation resources and integrate with other energy systems will be significantly improved.

For the smart interaction of clean power grid, the current grid has achieved a high level of intelligent dispatching, but the source-network-load interaction is relatively weak. In the future, with the increase of the penetration rate of new energy and the full response of demand-side resource, these two indicators will achieve great improvement.

For the low carbon and economy of clean power grid, as the grid-connected installed capacities and power generation scales of wind power, solar power, hydropower and nuclear power continue to increase, the climate friendliness of the grid will be significantly improved. With the development of multi-energy integration and the improvement of the intelligent level of power grid management and operation, the system operation will be more efficient and the level of comprehensive energy utilization will gradually increase. At present, the non-hydro renewable energy utilization, clean energy utilization, line loss rate, and transmission and distribution asset utilization are at a relatively good level. In the future, as the penetration rate of new energy continues to increase, the three indicators may fall within a certain range. 
For the flexibility of clean power grid, the current indicators of flexible power supply, demand-side response, grid operation flexibility, and electricity marketization are relatively weak. To support the development of a high proportion of clean energy, these indicators need to be significantly improved in the future.

In general, in order to promote the development of a

\section{Acknowledgements}

All the authors acknowledge the financial supports from Science and Technology Project of State Grid of Corporation of China titled with "Research on strategic planning and market mechanism of energy green transformation with re-electrification as the core".

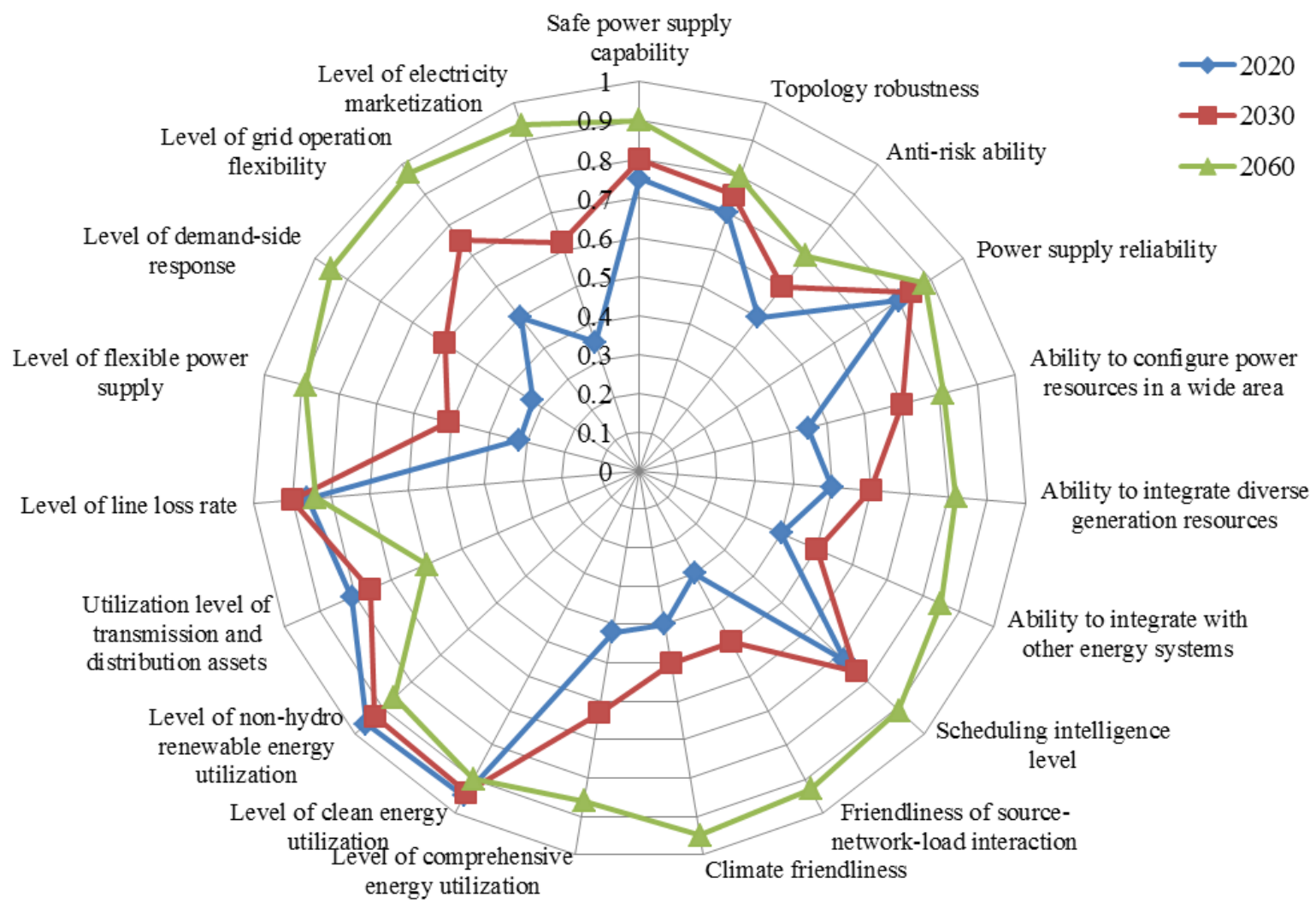

Figure 1. Radar chart of the evaluation of China's clean power grid development model in the medium and long term

high proportion of clean energy, it is necessary to focus on greater breakthroughs in the security, interconnection, smart interaction, flexibility of clean power grid, and to properly handle the declines of the indicators of lowcarbon and economy such as the non-hydro renewable energy utilization and the transmission and distribution asset utilization.

\section{Conclusion}

Oriented to the development of a clean power grid that supports the development of a high proportion of clean energy, the paper proposes a set of clean power grid development evaluation indicators including 5 first-level indicators and 19 second-level indicators. Taking China's power grid as an example, the radar chart analysis method is used to evaluate and analyze the characteristics of the medium and long-term development mode of the clean power grid and the relative change trends of specific indicators. Finally, the paper discusses the key links and potential problems that need attention in the development of the clean power grid.

\section{References}

1. Jinping Xi. Xi Jinping's speech at the general debate of the 75th UN General Assembly. (http://www.xinhuanet.com/politics/leaders/202009/22/c_112652764 7.htm.)

2. J. M. Alemany, B. Arendarski, P. Lombardi, et al. Accentuating the renewable energy exploitation: Evaluation of flexibility options[J]. International Journal of Electrical Power \& Energy Systems, 2018, 102: 131-151.

3. Bin Wang, Guangyu He, Shengwei Mei, and et al. Construction method of smart grid's assessment index system. Automation of Electric Power Systems, 2011, 35(23): 1-5.

4. $\mathrm{Xu}$ Wang, Junhui Huang, Jian Tan, and et al. Quantitative assessment on the world-class of the key indicators of grid evaluation. Proceedings of the CSU-EPSA, 2016, 28(2): 80-84.

5. G. Liu, M. Li, B. Zhou, et al. General indicator for techno-economic assessment of renewable energy resources[J]. Energy conversion and management, 2018, 156: 416-426. 
6. Shiwei Yu, Yali Zheng, and Longxi Li. "A comprehensive evaluation of the development and utilization of China's regional renewable energy."

Energy Policy 127 (2019): 73-86. 